\title{
PERFECT RINGS FOR WHICH THE CONVERSE OF SCHUR'S LEMMA HOLDS
}

\author{
A. Haily and M. Alaoui
}

\begin{abstract}
If $M$ is a simple module over a ring $R$ then, by the Schur's lemma, the endomorphism ring of $M$ is a division ring. However, the converse of this result does not hold in general, even when $R$ is artinian. In this short note, we consider perfect rings for which the converse assertion is true, and we show that these rings are exactly the primary decomposable ones.
\end{abstract}

\section{Introduction}

Let $M$ be a module over a ring $R$. If $M$ is simple, then the Schur's lemma states that $\operatorname{End}_{R}(M)$ is a division ring (a skew field). The converse of this statement is false. For example, if $R$ is an integral (commutative) domain which is not a field, then its quotient field $Q$, considered as an $R$-module, is not simple, although $\operatorname{End}_{R}(Q) \cong Q$ is a division ring.

For an example in the artinian case, one can take: $R=\left(\begin{array}{cc}K & K \\ 0 & K\end{array}\right)$, the ring of upper triangular $2 \times 2$ matrices over a field $K$. Then for the $R$-module $M=R e$, where $e=\left(\begin{array}{ll}0 & 0 \\ 0 & 1\end{array}\right)$, we have $\operatorname{End}_{R}(M) \cong K$, but $M$ is not simple.

Definition 1.1. We shall say that a ring $R$ has the CSL property (abreviation of: Converse of the Schur's Lemma), or that $R$ is a CSL-ring, if every module is simple whenever its endomorphism ring is a division ring.

The CSL property, has been studied by some authors. In [4], Ware and Zelmanowitz, considered modules with simple endomorphism ring over a commutative ring. From their results, it can be shown that a commutative ring $R$ is a CSL-ring iff every prime ideal of $R$ is maximal. In [3] some classes of noncommutative von Neumann regular rings with the CSL property has been studied.

2000 Mathematics Subject Classification. 16D60, 16K40.

Key words. Schur's lemma, perfect rings, simple module, uniform module. 
The full class of CSL-rings seems to be very hard to characterize, the present note deals with perfect CSL-rings. Our main result is:

Theorem 1.2. For a perfect ring $R$, the following assertions are equivalent:

(i) Every R-module with semiprime endomorphism ring is semisimple.

(ii) Every $R$-module with von Neumann regular endomorphism ring is semisimple.

(iii) $R$ is a CSL-ring.

(iv) $R$ is isomorphic to a finite product of primary rings.

\section{Preliminaries and notations}

(For the terminology and notations used here we refer to [1], [2].)

Throughout this paper, all rings are associative with identity, and all modules are left unitary modules. If $M$ is a module over a ring $R$, the endomorphism ring of $M$ is denoted by $\operatorname{End}_{R}(M)$. The socle of $M$, i.e. the sum of all simple submodules of $M$, is denoted by $\operatorname{Soc}(M)$.

A ring $R$ is said to be perfect if it is left and right perfect. Over a perfect ring, every nonzero module has a maximal and a simple submodule.

A ring $R$ is said to be primary, if the factor ring $R / J(R)$, where $J(R)$ denotes the Jacobson radical of $R$, is simple artinian. Any primary left or right perfect ring is isomorphic to a full matrix ring over a local ring [2].

A right or left perfect ring $R$ is said to be primary decomposable, if it is isomorphic to a (finite) product of primary rings. It can be shown that $R$ is primary decomposable, if and only if, every idempotent which is central modulo the Jacobson radical is central.

A ring $R$ is said to be von Neumann regular (abbreviated VNR), if for every $x \in R$ there exists $y \in R$ such that $x y x=x$. An important example of a VNR ring is the endomorphism ring of a semisimple module.

\section{The proofs}

(i) $\Rightarrow$ (ii) is obvious since every VNR ring is semiprime.

(ii) $\Rightarrow$ (iii). If $\operatorname{End}_{R}(M)$ is a division ring, then it is VNR. So $M$ is semisimple by hypothesis. Since $M$ is indecomposable, it is therefore simple.

(iv) $\Rightarrow$ (i). It is easy to see that any direct product of a finite number of rings verifying (i) has this property. Hence to show that (iv) implies (i), it suffices to show that every perfect primary ring verifies (i). Let $R$ be such a ring. If $M$ is any nonzero $R$-module, then $M$ has a maximal submodule $N$, and a simple submodule $S$. Since $R$ is primary, $R$ has a 
unique isomorphism class of simple modules, so there exists an $R$-module isomorphism $\sigma: M / N \rightarrow S$. If $\pi: M \rightarrow M / N$ and $\imath: S \rightarrow M$ denote respectively the canonical surjection and the canonical injection, then $u=\imath \circ \sigma \circ \pi$ is a nonzero endomorphism of $M$ such that $u(N)=0$ and $u(M) \subset S$.

Now suppose that $M$ is not semisimple, then $M$ contains a proper essential submodule $E$ which is contained in a maximal submodule $N$. By what has been proved previously, there exists a nonzero $u \in \operatorname{End}_{R}(M)$ such that $u(N)=0$ and $u(M) \subset \operatorname{Soc}(M)$. Since $E$ is essential, we have $\operatorname{Soc}(M) \subset E$ and then $u(\operatorname{Soc}(M)) \subset u(N)=0$. Now for every $v \in \operatorname{End}_{R}(M),(u \circ v \circ u)(M) \subset(u \circ v)(\operatorname{Soc}(M)) \subset u(\operatorname{Soc}(M))=0$. This proves that $u \circ v \circ u=0$ for every $v \in \operatorname{End}_{R}(M)$; so that $\operatorname{End}_{R}(M)$ is not semiprime.

(iii) $\Rightarrow$ (iv). To prove this implication, we need a preliminary result.

Lemma 3.1. Let $M$ be a finitely generated module over a perfect ring $R$. Suppose that $\operatorname{Hom}_{R}(N, \operatorname{Soc}(M))=0$ for every nonsimple submodule $N$ of $M$. Then $\operatorname{End}_{R}(M)$ is a division ring.

Proof: Suppose that $\operatorname{End}_{R}(M)$ is not a division ring, then there exists $u \in \operatorname{End}_{R}(M)$ such that $u$ is nonzero and noninvertible. Since $M$ is finitely generated over a perfect ring, $u$ is not injective. Let $N$ be a submodule of $M$ such that $\operatorname{Ker} \subset N$ and $N / \operatorname{Ker} u$ is simple. If $v=\left.u\right|_{N}$ denotes the restriction of $u$ to $N$, then $\operatorname{Im} v \cong N / \operatorname{Ker} v$ so $\operatorname{Im} v$ is simple. Thus $\operatorname{Im} v \subset \operatorname{Soc}(M)$. This proves that $\operatorname{Hom}(N, \operatorname{Soc}(M)) \neq 0$.

We are now going to prove the implication (iii) $\Rightarrow$ (iv). Suppose on the contrary that $R$ is a CSL-ring which is not primary decomposable. Then there exists an idempotent $e \in R$ central modulo $J=J(R)$ but not central. Either $R(1-e) R e \neq 0$ or $R e R(1-e) \neq 0$. Without loss of generality, we can suppose that $R(1-e) R e \neq 0$. Since $R(1-e) R e \neq$ $J(1-e) R e$, we can pick an element $x \in R(1-e) R e \backslash J(1-e) R e$, and consider the left ideal $I$ maximal with respect to:

$$
J(1-e) R e \subset I \subset R e \text { and } x \notin I .
$$

Then, the module $M=R e / I$ is finitely generated with simple socle equal to $S=R x+I / I$. Since $J(1-e) R e \subset I$, we have $J(1-e) M=0$. Hence $(1-e) M \subset S$. On the other hand, $e R \subset R e+J$, thus $e R(1-e) R e \subset$ $J(1-e) R e$, implying $e S=0$.

Now let $N$ be a submodule of $M$ such that $\operatorname{Hom}_{R}(N, S) \neq 0$ and $u: N \rightarrow S$ a nonzero homomorphism. We have $u(N)=S$ and $u((1-$ $e) N)=(1-e) S \neq 0$. Since $(1-e) N \subset S$, then $u(S) \neq 0$. Consequently Ker $u=0$ and $u$ is therefore an isomorphism. So $N$ is necessarly simple. 
By Lemma 3.1, $\operatorname{End}_{R}(M)$ is a division ring. Since $R$ is a CSL-ring, $M$ is simple. So $M=S$ and $e M=e S=0$, a contradiction.

\section{References}

[1] F. W. Anderson And K. R. Fuller, "Rings and categories of modules", Graduate Texts in Mathematics 13, Springer-Verlag, New York, 1974.

[2] C. FAith, "Algebra. II. Ring theory", Grundlehren der Mathematischen Wissenschaften 191, Springer-Verlag, Berlin, 1976.

[3] Y. Hirano And J. K. PARK, Rings for which the converse of Schur's lemma holds, Math. J. Okayama Univ. 33 (1991), 121-131.

[4] R. Ware And J. Zelmanowitz, Simple endomorphism rings, Amer. Math. Monthly 77 (1970), 987-989.

Département de Mathématiques

Faculté des Sciences

B.P. 20, El Jadida

Morocco

E-mail address: haily@ucd.ac.ma

E-mail address: alaoui_m@ucd.ac.ma

Primera versió rebuda el 6 de juny de 2000, darrera versió rebuda el 31 d'octubre de 2000. 